\title{
LA RESPONSABILIDAD SOCIAL, CORPORATIVA COMO REFUERZO DE LA ESTRATEGIA Y LA COMPETITIVIDAD EMPRESARIAL
}

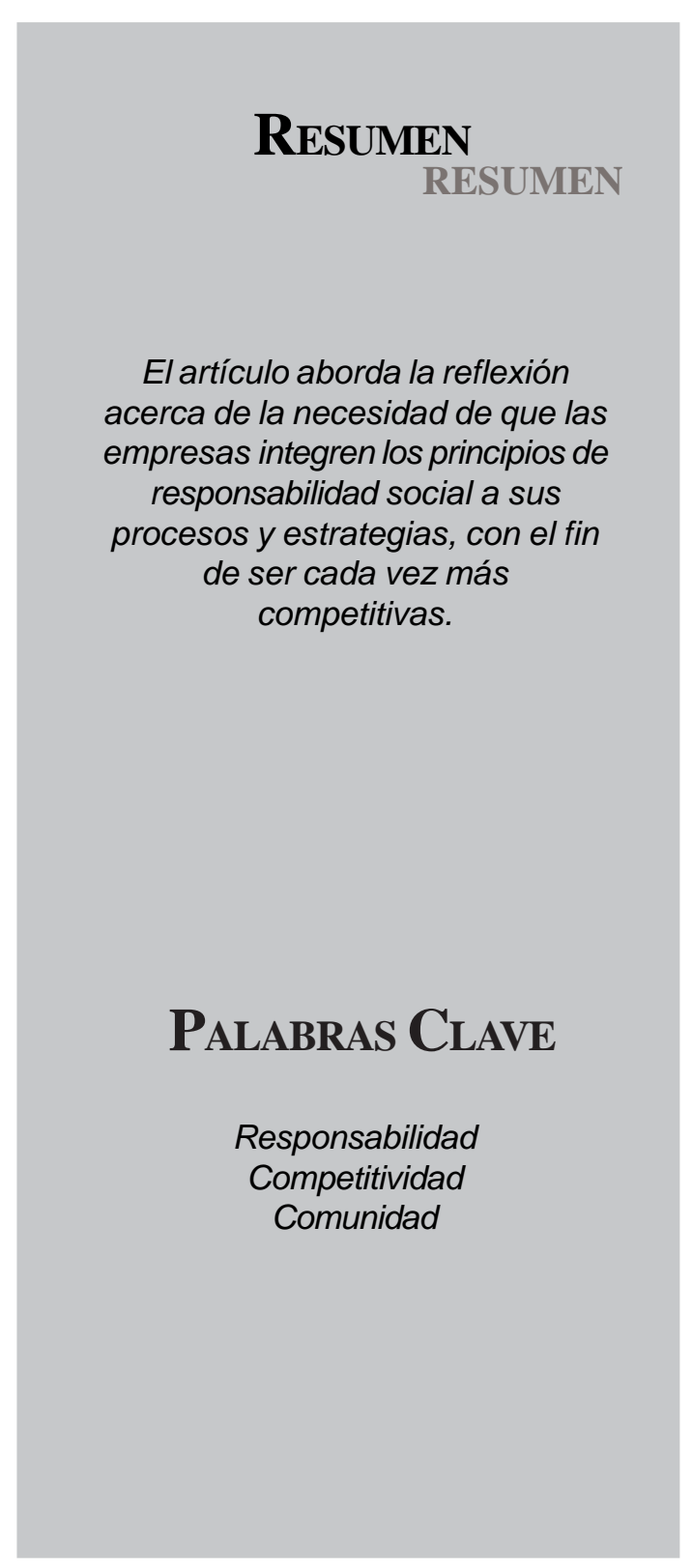

Sabin Azua*

$\mathbf{E}$

stamos asistiendo a una dinámica de socialización del concepto de responsabilidad social corporativa. Las numerosas iniciativas que se han puesto en marcha a nivel internacional están generando un caldo de cultivo importante para el desarrollo de prácticas de mejora de la gestión de dicha responsabilidad por parte de un número importante de organizaciones. La Global Reporting Initiative, el Libro Blanco para el Fomento de la Responsabilidad Social de la Comisión, las Líneas Directrices de la OCDE para empresas multinacionales, etc., son algunas iniciativas que generan un marco para la toma de decisiones a nivel individual.

Como ocurre con otras modas o tendencias, hay que señalar que las empresas no parten de cero en este campo, por mucho que no hayan tomado decisiones de RSC de forma consciente o conformando parte de un esquema. Las empresas que son competitivas tienen, con carácter general, elementos de

\footnotetext{
* Socio, director de B + I Strategy

Artículo publicado en:Revista APD, Monográfico Responsabilidad Social Corporativa Septiembre 2006 $\mathrm{N}^{\circ} 212$.
}

Este artículo fué entregado el 11 de octubre de 2007 y su publicación aprobada por el Comité Editorial el 29 de novimebre de 2007 
gestión de responsabilidad sin los cuales hubiesen dejado de existir hace tiempo. No obstante, creo que es muy útil que las organizaciones tomen consciencia de la necesidad de integrar los principios de la RSC en sus procesos de reflexión y praxis estratégicas.

En mi opinión, la primera responsabilidad que tienen las empresas es cumplir con sus principios básicos: desarrollar productos y servicios que sean útiles para la comunidad (representada por sus clientes), que genere empleo de calidad y respete el desarrollo profesional y personal de sus trabajadores, crear suficiente riqueza de forma que se consolide el proyecto empresarial en el largo plazo; todo ello dentro del estricto respeto a la/s Comunidad/es en la/s que desarrolla su actividad, contribuyendo al bien común, de acuerdo con lo expresado por el Profesor Miguel Ángel Gallo en su libro 'La Responsabilidad Social de la Empresa'.

Yo no creo que existan empresas competitivas en el largo plazo que no sean empresas comprometidas con los principios de la RSC. Las empresas que manifiestan un fuerte compromiso de fomento de la competitividad son aquellas que contribuyen al progreso económico, social y medioambiental a lo largo del tiempo, desde un profundo respeto por los derechos humanos en todos sus ámbitos geográficos de actuación, tienen una fuerte interrelación con el entorno competitivo en el que trabajan.

Asimismo, son organizaciones que hacen del fomento del capital humano y del intercambio de conocimiento una constante de actuación, mostrándose honestas, transparentes y respetuosas con la legalidad vigente, aplicando las mejores prácticas de buen gobierno, comercializando 'productos éticos', etc.

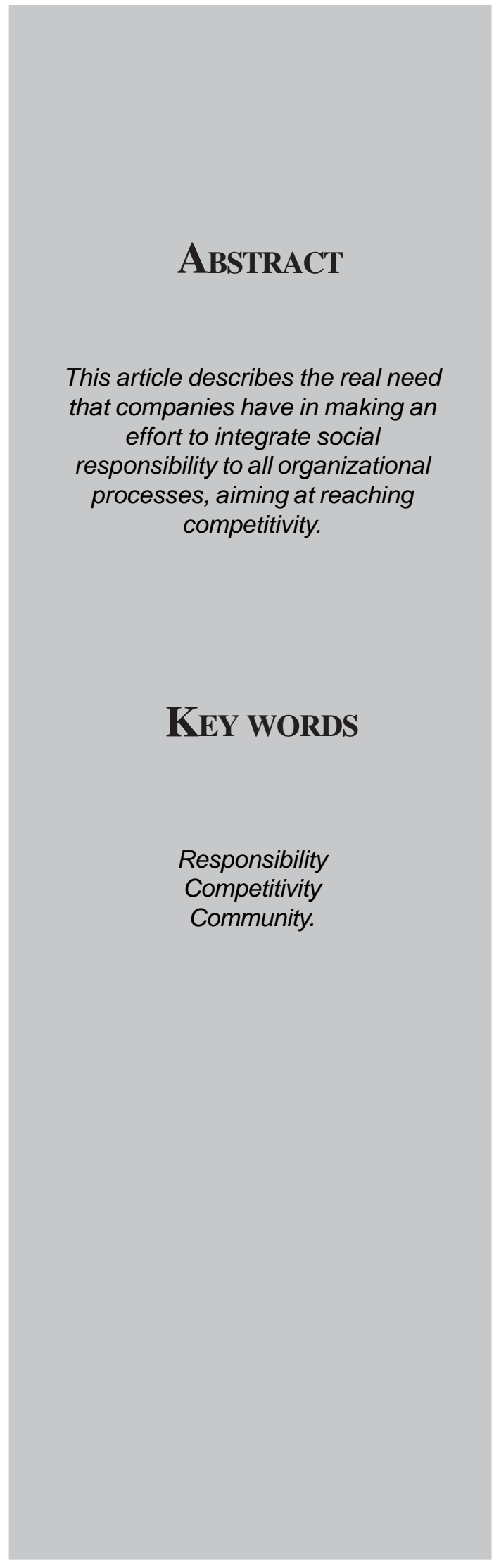

This article describes the real need effort to integrate social responsibility to all organizational processes, aiming at reaching competitivity.

$$
\begin{aligned}
& \text { Responsibility } \\
& \text { Competitivity } \\
& \text { Community. }
\end{aligned}
$$


Creo que las enormes diferencias sociales en los países avanzados y semidesarrollados del mundo, así como el abismal infradesarrollo de amplias zonas del planeta, fuerzan a que las empresas - en el marco de los principios de Responsabilidad Social Corporativa - adopten estrategias y prácticas de actuación encaminadas a favorecer la mejora de las condiciones de vida de la parte baja de la pirámide. En este artículo quiero recoger las enseñanzas del Profesor C.K. Prahalad en su libro 'The Fortune at the Bottom of the Pyramid: Erradicating Poverty Through Profits', que nos estimula a adoptar estrategias innovadoras para generar negocios que mejoren las condiciones de vida de estratos sociales desfavorecidos generando mejoras en la propia competitividad empresarial.

En el marco de este proceso establecido por el Profesor Prahalad, debemos generar negocios compatibles con las necesidades de la base de la pirámide (no pensemos solamente en el tercer y cuarto mundo, sino en las zonas desfavorecidas de nuestro mundo desarrollado), cuyas características de mercados son: pequeñas unidades o dosis de producto, muy bajo margen por unidad, elevado volumen y alta rentabilidad por capital empleado. La atención de estos mercados de forma eficiente y responsable implica, entre otros, los siguientes elementos: focalización en el precio de los productos y servicios, soluciones a medida altamente innovadoras y generalizables en diferentes entornos culturales y/o geográficos, incidencia en la recuperación de materiales, preferencia de la funcionabilidad sobre la estética, la formación a los consumidores y sostenibilidad en la atención de estas y otras necesidades futuras que puedan utilizar las plataformas generadas, etc.

Como cierre me gustaría señalar que la RSC no debe ser una actividad para generar una memoria de sostenibilidad o de buen gobierno, sino una parte esencial de la estrategia competitiva de cualquier organización. Como dijo Lindsay Kemp ante el Foro de Porto Alegre: 'Quitémonos el maquillaje, mostrándonos como somos cumpliremos con nuestras obligaciones frente al resto del mundo'. 


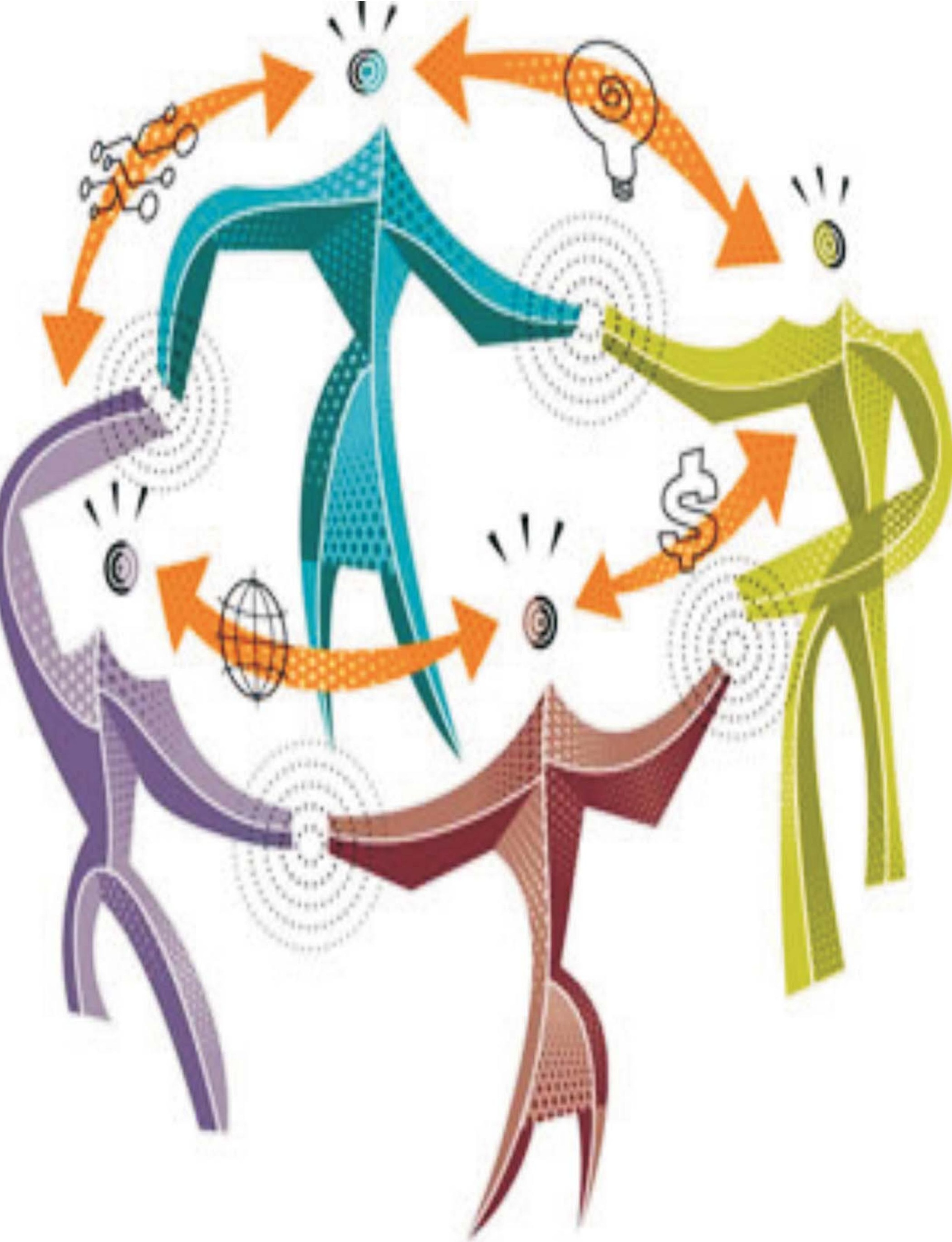




\section{LA EMPRESA \\ PARTICIPATIVA Y LAS TIC}

Isabel Fernández Rodríguez*

\section{RESUMEN}

RESUMEN

El artículo centra su reflexión

en la capacidad de generar

participación en la empresa, tarea en

al cual se implica la presencia de las tecnologías de información y

comunicación y se relevan algunas

herramientas específicas que

favorecen la participación en cuatro

formas: en la información y la

comunicación, en la definición de

objetivos, en la toma de decisiones y

en la creación de conocimiento.

\section{Palabras Clave}

Empresa participativa Generar participación

Tecnologías de información y comunicación
En los últimos tiempos no paramos de oír hablar de empresas más participativas como modelos de organizaciones favorecedoras de prácticas innovadoras en nuestro entorno. Tal y como señala D. Santiago García Echevarría', 'solamente cuando se disponga de instituciones y organizaciones flexibles es cuando puede innovarse. La innovación no sale de instituciones rígidas o jerarquizadas sino de instituciones empresariales con gran rapidez de adaptación y flexibilidad'.

Hablamos de organizaciones más innovadoras como factor clave para poder competir en un entorno global donde diferenciarnos de nuestros competidores, también globales, requiere de empresas que sean capaces de ofrecer bienes y servicios diferentes a los que el resto de empresas ofrecen.

Pero vayamos al grano: ¿qué entendemos por empresas participativas? Puesto que participar es algo que, en mayor o menor medida, lo hacen todas las personas de una organización, la pregunta más precisa sería ¿qué entendemos por empresas más participativas?

\footnotetext{
* Colaboradora del Cluster del Conocimiento.

1 García Echavarría, S. La revolución institucional en la empresa. Cuadernos de Empresas y Humanismo.
} 
En nuestra opinión se trata de un concepto muy ambiguo dada la diversidad de enfoques desde los que se analiza. De cara a no perdernos en debates dialécticos, nosotros defendemos una definición en su dimensión más amplia en la que asumimos que 'la participación se refiere a todos los métodos que permiten a los trabajadores influir en la gestión de la empresa'. Se trataría, por lo tanto, del grado de implicación y de compromiso que las personas de una organización adquieren en el proceso de toma de decisiones ${ }^{2}$.

Por otra parte, entendemos que una cosa es generar la capacidad de participar y otra muy diferente es generar el deseo de participar por parte de las personas. En este artículo vamos en centrarnos precisamente en el primer aspecto: generar la capacidad de participar.

Para ello, tomamos como base de partida las formas de participación que definen Ayestarán, S. et al [2006] en su publicación 'Rumbo a la innovación: Trabajo en equipo y cambio cultural en las organizaciones'. En su investigación, distinguen cuatro formas de participación:

- Participación en la información y comunicación, forma en la que se exige transparencia de la información y acceso a la misma de las personas que conforman la organización.

- Participación en la definición de objetivos, donde la aportación de opiniones por parte de las personas así como el conocimiento individual y grupal de la metodología PDCA [Planificar, Hacer, Evaluar y Ajustar] resultan aspectos clave para que la participación se pueda dar.

2 Cuesta, I., y Fernández, I. «Modelo Vasco de Empresas Participativas». (2001).

\section{Abstract}

This article makes a reflection on the capacity to generate company participation, a task which implies the presence of information and communication technologies, and some specific tools

which favor participation in three different ways are also described, taking into account information and communication, the definition of clear aims, decision making and creation of knowledge.

\section{KEY WORDS}

Participative company Generating participation Information and Communication technologies. 
- Participación en la toma de decisiones, individual y/o colectivamente, donde necesariamente las personas deben conocer el despliegue de el/los proceso/s en los que toman parte, de cara a la toma de decisión más idónea.

- Participación en la creación de conocimiento, donde el mero hecho del intercambio de información no es suficiente: ha de darse además una coordinación, una integración de ideas, perspectivas y experiencias que faciliten el descubrimiento de una nueva idea.

Retomando la frase de D. Santiago García Echevarría con la que comenzamos esta reflexión 'La innovación no sale de instituciones rígidas o jerarquizadas sino de instituciones empresariales con gran rapidez de adaptación y flexibilidad' nos percatamos de que las TIC [Tecnologías de la Información y Comunicación] juegan un papel fundamental. La intensidad con que se ha dado la llamada revolución de las TIC ha producido un impacto directo en las formas de organizarse, han permitido que el mundo pueda no continuar siendo un lugar compuesto de compartimentos estancos sino un lugar donde las conexiones entre individuos y organizaciones sean más posibles, más ágiles y más flexibles; en definitiva, las organizaciones planas son organizaciones que favorecen la actuación en red y esto, es posible gracias a las TIC.

Y a raíz de estas reflexiones nos preguntamos, ¿tenemos hoy en día a nuestra disposición herramientas TIC que ayuden a generar capacidad de participar en la gestión de las organizaciones de una manera flexible? Nosotros pensamos que sí. Hoy en día contamos con herramientas que nos permiten fomentar la participación en la información y comunicación, en la definición de objetivos [estratégicos y tácticos], en la toma de decisiones y en la creación de conocimiento, siendo esta última desde nuestro punto de vista, el gran reto de nuestra sociedad.

A continuación, señalamos una serie de soluciones [destacamos sólo algunas de ellas] que, desde nuestro punto de vista, usadas correctamente, pueden favorecer las cuatro formas de participación que hemos mencionado:

- Weblog o blog: según definición de wikipedia, 'es un sitio web periódicamente actualizado que recopila cronológicamente textos o artículos de uno o varios autores, apareciendo primero el más reciente, donde el autor conserva siempre la libertad de dejar publicado lo que crea pertinente. Mediante un formulario se permite, a otros usuarios de la web, añadir comentarios a cada entrada, pudiéndose generar un debate alrededor de sus contenidos'. Aplicado a empresas, puede fomentar la gestión de conocimiento de 'abajo a arriba', facilita la coordinación de equipos dispersos geográficamente y fortalece la cultura interna de la empresa.

- ERP [Enterprise Resource Planning]: se trata de un conjunto de aplicaciones que facilitan la gestión y la integración de los distintos procesos que intervienen en la cadena de valor de la empresa. Proveen de herramientas que permiten compartir información entre los procesos y comunicarse entre ellos, de manera que las personas que componen la empresa disponen de información precisa para la toma de decisiones, en todos los niveles.

- KM [Knowledge management]: herramienta que proporciona sistemas que facilitan la gestión documental de la empresa así como su clasificación y búsqueda de manera rápida y sencilla. 
Asimismo, puede tener otras funcionalidades como son foros virtuales que permiten compartir experiencias entre las personas que conforman la organización.

- Business Intelligence [Inteligencia empresarial o de negocio]: según definición de wikipedia, 'conjunto de estrategias y herramientas enfocadas a la administración y creación de conocimiento mediante el análisis de datos existentes en una organización o empresa. Este conjunto de herramientas y metodologías tienen en común las siguientes características: accesibilidad a la información, apoyo en la toma de decisiones y orientación al usuario final. Se trata por tanto, del proceso de análisis de datos de la empresa para poder extraer conocimiento de ellos. Con BI se puede: crear una base de datos de clientes, prever ventas y devoluciones, compartir información entre diferentes departamentos, mejorar el servicio al cliente'.

Sin embargo queremos destacar que el reto no sólo consiste en identificar las herramientas más adecuadas para el tipo de participación que queremos fomentar en nuestra organización. Es necesario pero no suficiente: resulta clave conocer el impacto que esa herramienta va a provocar en las formas de trabajo de las personas que componen la organización. ¡Cuántas empresas han realizado inversiones en herramientas que no se están aprovechando adecuadamente!

Y tú, ¿conoces lo que las herramientas TIC pueden hacer por tu organización para conseguir una mayor participación de las personas que la componen? ¿Conoces las implicaciones que puede traer consigo su implantación, el impacto en las formas de trabajo y en las personas? 